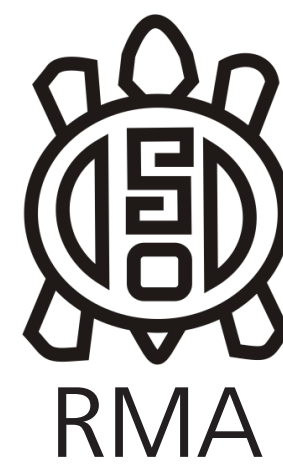

Dossier

\title{
Una aproximación estadística al estudio de los cambios entésicos empleados para evaluar diferencias sexuales en restos humanos
}

\author{
A statistical approach to entheseal changes analyses to infer sexual \\ differences in human remains \\ Bárbara Mazza
}

Instituto Nacional de Antropología y Pensamiento Latinoamericano - CONICET. E-mail: barbara_mazza@yahoo.com.ar

\begin{abstract}
Resumen
El estudio de los cambios entésicos en poblaciones pasadas ha sido abordado a través de varios análisis estadísticos. Sin embargo, esta variabilidad metodológica dificulta la comparación de los resultados. El objetivo de este trabajo es presentar y discutir los resultados de una serie de análisis estadísticos comúnmente adoptados para el estudio de diferencias sexuales en los cambios entésicos con el fin de sugerir el método más eficaz. Para ello se aplicaron los siguientes análisis: distribución de frecuencias, cálculo de medias aritméticas y desvíos estándar, prueba de chi-cuadrado, U de Mann-Whitney, $t$ de Student, correlación simple y parcial de Spearman y modelos lineales generalizados. La muestra analizada está integrada por 40 individuos de ambos sexos provenientes de poblaciones cazadoras-recolectoras del humedal del Paraná inferior. La mayoría de los análisis mostraron diferencias significativas entre ambos sexos. Sin embargo, debido a la etiología multifactorial de los cambios entésicos, se recomiendan aquellas pruebas estadísticas donde se puedan ingresar covariables, como ser las correlaciones parciales o los modelos lineales generalizados.
\end{abstract}

Palabras claves: análisis bivariados; correlaciones parciales, modelos lineales generalizados; entesis; diferencias sexuales.

\begin{abstract}
Entheseal changes have been analyzed through several statistical approaches. This methodological variability hinders cross-comparisons. The aim of this work is to present and discuss the results of a statistical set of analyses commonly applied in the study of sexual differences in entheseal changes with the final objective of suggesting the most effective method. For this purpose, it was applied the following statistical tests: frequency distribution, arithmetic mean and standard deviation, Chi-square test, Mann-Whitney $U$ test, $t$-Student, Spearman simple and partial correlations and generalized linear models. The sample investigated was composed by 40 adult individuals of both sexes from hunter-gatherer populations from lower Paraná wetlands. Most statistical tests showed significant differences between both sexes. However, since entheseal changes have a multifactorial etiology, it is recommended to apply statistical tests that control the effect of some variables, such as partial correlations and generalized linear models.
\end{abstract}

Keywords: bivariate analysis; partial correlations; generalized linear models; entheses; sexual differences.

Los cambios entésicos son modificaciones en la morfología ósea de las zonas de inserción de músculos, ligamentos y tendones, denominadas entesis. Estos cambios son provocados tanto por estrés mecánico como por factores de índole inflamatoria, degenerativa, endócrina, metabólica o traumática (Resnick y Niwayama 1983). Debido a esta etiología multifactorial, la incorporación de los cambios entésicos como variable para estudiar aspectos culturales en poblaciones pasadas requiere la inclusión de varios factores y, por lo tanto, de un minucioso análisis estadístico.

La mayoría de los trabajos que analizan cambios entésicos en poblaciones pasadas, generalmente con fines de evaluar diferencias sexuales y/o etarias dentro de una población, emplean análisis uni y bivariados de los datos, es decir análisis de frecuencias, medias aritméticas y empleos de test estadísticos tales como: Chi-cuadrado, U de Mann-Whitney y $t$ de Student, (e.g. Hagaman 2009; Peterson 1998). Estos análisis permiten evaluar si existen diferencias significativas entre dos niveles de un mismo factor, por ejemplo diferencias entre el sexo femenino y masculino.

En los últimos años, dado el conocimiento multifactorial que afecta a los cambios entésicos, se han incorporado 
análisis de correlaciones simples y parciales de Spearman (e.g.Weiss 2007), así como de modelos lineales generalizados (de aquí en más MLG; Henderson y Nikita 2015; Nikita 2014). Este último análisis comenzó a ser recomendado para estudiar manifestaciones óseas con etiología multifactorial, ya que permite analizar y controlar los efectos de más de una variable al mismo tiempo (McCullagh y Nelder 1989).

La variabilidad metodológica mencionada dificulta la comparación de los resultados y la estandarización de enfoques metodológicos. Por lo tanto, el objetivo de este trabajo es presentar y discutir los resultados de una serie de análisis estadísticos comúnmente adoptados para el estudio de diferencias sexuales en los cambios entésicos con el fin de sugerir el método más adecuado. Para ello se analiza una muestra de 40 individuos de ambos sexos provenientes de poblaciones cazadorasrecolectoras del humedal del Paraná inferior. Se aplican métodos estadísticos uni y bivariados y modelos lineales generalizados para evaluar diferencias sexuales en los cambios entésicos, como así también la influencia de la edad y el tamaño corporal sobre la morfología de la entesis.

\section{Materiales y Métodos}

La muestra está compuesta por 40 individuos adultos de ambos sexos (11 femeninos, 29 masculinos), provenientes de los siguientes sitios arqueológicos localizados en la región del humedal del Paraná inferior (Argentina):

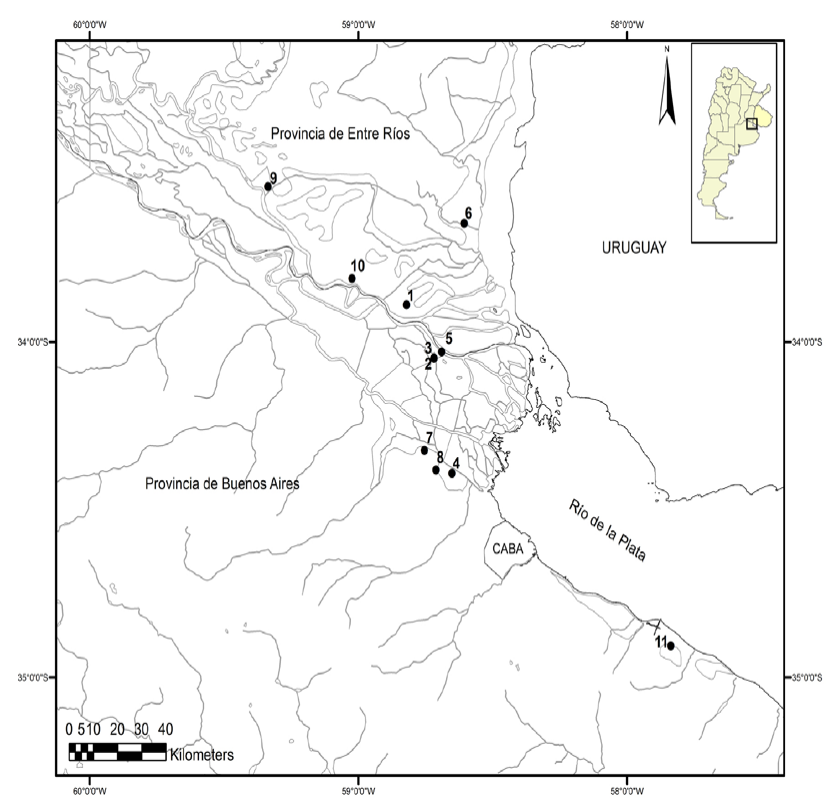

Figura 1. Ubicación de los sitios arqueológicos analizados en este trabajo. 1: Túmulo II del Brazo Largo; 2: Arroyo Marieta; 3: Arroyo Los Tigres; 4: Arroyo Sarandí; 5: El Cerrillo; 6: Cerro Lutz; 7: El Cazador sitio 3; 8: Garín; 9: Paraná Ibicuy 1 / La Argentina; 10: Escuela 31; 11: Los Talas.

Figure 1. Location of the archaeological sites analyzed in this work. 1: Túmulo II del Brazo Largo; 2: Arroyo Marieta; 3: Arroyo Los Tigres; 4: Arroyo Sarandi; 5: El Cerrillo; 6: Cerro Lutz; 7: El Cazador sitio 3; 8: Garín; 9: Paraná Ibicuy 1 / La Argentina; 10: Escuela 31; 11: Los Talas.
Túmulo II del Brazo Largo $(n=6)$, Arroyo Marieta $(n=7)$, Arroyo Los Tigres $(n=3)$ (Mazza 2015), Arroyo Sarandí $(n=3)$, El Cerrillo $(n=2)$ (Lothrop 1932), Cerro Lutz $(n=$ 9; Mazza 2010), El Cazador sitio 3 ( $n=1$; Loponte y Acosta 2011), Garín ( $n=1$; Loponte 2008), Paraná Ibicuy 1/La Argentina ( $n=3$; Acosta y Mazza 2016; Caggiano et al. 1978), Los Talas ( $n=3$; Vignati 1960), Escuela $31(n=2$; Loponte et al. 2015) (Figura 1). Todos los sitios corresponden a la última fase del Holoceno tardío (2000700 años AP).

La determinación sexual se realizó siguiendo los delineamientos establecidos por Buikstra y Ubelaker (1994) para el cráneo y coxales, como así el método de Phenice (1969) para estos últimos. Para la estimación etaria se tuvo en cuenta los cambios en la sínfisis púbica (Brooks y Suchey 1990) y en superficie auricular del ilion (Lovejoy et al. 1985), y el grado de obliteración de las suturas craneales (Meindl y Lovejoy 1985) y de las epífisis (Scheuer y Black 2000). De esta forma, se construyeron dos rangos etarios: $20-40(n=17)$ y mayores de 40 años $(n=23)$. Se decidió dividir la muestra en dos rangos etarios porque, en primer lugar, de esta manera cada categoría tiene un tamaño de muestra aceptable para realizar comparaciones estadísticas, que de lo contrario (si se fragmenta la muestra en mayores rangos etarios), la cantidad individuos en cada uno de ellos sería muy baja. En segundo lugar, el punto de quiebre de 40 años es apropiado ya que luego de esta edad se manifiesta una mayor pérdida ósea y cambios osteoporóticos que impactan en la morfología de las entesis (Niinimäki 2012).

Como proxy del tamaño corporal, se tomaron las longitudes máximas y anchos epifisiales de todos los huesos largos, según los criterios establecidos por Buisktra y Ubelaker (1994). Para ello se empleó una tabla osteométrica y un calibre digital con una precisión de 0.01 mm (marca Baker). En primer lugar, se calculó el error intraobservador para cada variable métrica, a través del estadístico ANOVA de medidas repetidas $(p<0.10)$ y del índice de correlación intraclase (ICC<0.60) (Zar 2010). Para ello se tomaron tres veces las mediciones con una separación de diez días entre ellas. Los resultados de replicación no arrojaron diferencias significativas entre las mediciones. Entonces, una vez corroborada la ausencia de errores intraobservador, se realizó una prueba $t$ apareada $(\alpha=0.050)$ para evaluar la presencia de diferencias significativas entre la lateralidad derecha e izquierda con el objetivo de conocer si era pertinente la combinación de ambas lateralidades para el cálculo del tamaño corporal. Debido a la ausencia de resultados significativos ( $p>0.050)$, se justificó la combinación de ambos lados. Luego, se calcularon los valores-z para cada medición con el fin de estandarizarlas y equipararlas (Zar 2010). Finalmente, se crearon variables compuestas para cada hueso largo por separado a partir del promedio de los valores-z de la longitud máxima y del ancho de las epífisis (Tabla 1; Henderson y Nikita 2015; Weiss 2003). En este 
trabajo, se decidió no aplicar fórmulas para la estimación del tamaño corporal (e.g. Auerbach y Ruff 2010; Raxter et al. 2006) debido a que la mayoría de ellas trabajan con varios elementos óseos de un mismo individuo. En la presente muestra, la mayoría de los esqueletos no cuentan con todos los elementos anatómicos. Su aplicación habría ido en detrimento de la cantidad de individuos analizados. Por otro lado, el uso de una sola medida para la aproximación del tamaño corporal no es recomendable debido a variaciones en el tamaño del esqueleto que pueden impactar en la biomecánica del sistema músculo-esqueletal (Henderson 2013a).

Se excluyeron del análisis aquellos individuos con señales patológicas o traumáticas que podrían haber influido en la morfología de las entesis (Henderson 2013b)

\section{El registro de los cambios entésicos}

Los cambios entésicos se reconocieron a través de modificaciones normales (i.e. no patológicas) en la morfología de la superficie de las entesis. Estas modificaciones se denominan robusticidad y reflejan una respuesta fisiológica ósea frente a la solicitud de músculos y ligamentos (Mariotti et al. 2007). En este sentido, la robusticidad es una categoría analítica que siempre está presente, aunque con distintos grados de expresión que dependen de varios factores (magnitud del estrés, sexo, edad, tamaño corporal, patologías, traumas, etc.). La robusticidad fue relevada según el estándar desarrollado por Mariotti et al.(2007), quienes registraron tres grados de desarrollo: $1=$ bajo y mediano, $2=$ fuerte y $3=$ muy fuerte.

Se analizó un total de 15 entesis pertenecientes a miembros superiores e inferiores (Húmero: pectoral mayor, dorsal ancho, deltoides y braquiorradial; Cúbito: tríceps, supinador y braquial anterior; Radio: bíceps, pronador redondo y membrana interósea; Fémur: glúteo mayor, vasto medial e iliopsoas; Tibia: cúadriceps y sóleo). Previo a su análisis, se evaluó el error intra observador en el registro de la robusticidad para cada entesis. Para ello, se relevaron tres veces los grados de robusticidad con una diferencia de diez días entre ellos. El estadístico utilizado en esta oportunidad fue el índice de Kappa de Cohen (K<0.60) (Aguado et al. 1990; Kramer et al. 1981). Todas las mediciones mostraron buenos porcentajes de coincidencia.

\section{Análisis estadísticos}

Si bien las entesis analizadas no presentan una distribución normal (test de Shapiro-Wilk, $p<0.050$ ) y por ende deberían aplicarse pruebas estadísticas no paramétricas, las variables ordinales pueden ser analizadas con estadísticos paramétricos cuando representan rasgos morfológicos de variación continua subyacente entre los distintos grados de expresión, tal como lo son los cambios entésicos (Nikita 2014; Robb 1998). Por lo tanto, en primer lugar, en este trabajo la presencia de diferencias sexuales en los cambios entésicos se exploró a través de análisis estadísticos univariados y descriptivos: distribución de frecuencias relativas entre los tres grados de robusticidad, cálculo de la media aritmética y desvío estándar.

Luego, se aplicaron cuatro análisis estadísticos bivariados con fines de evaluar su eficacia en detectar diferencias estadísticamente significativas entre ambos sexos ( $\alpha=$ 0.050). Se implementaron tres pruebas no paramétricas: Chi-cuadrado, U de Mann-Whitney y correlación de rango de Spearman, y una prueba paramétrica: $t$ de Student. Se compararon los valores $p$ de cada una de las pruebas, evaluando aquellas que arrojaban resultados similares y aquellas que diferían. Estos análisis nos permiten evaluar si existen diferencias significativas entre dos niveles de un mismo factor (en este trabajo diferencias entre el sexo femenino y masculino) con respecto a una variable cualitativa u ordinal (los cambios entésicos). Sin embargo, cada uno de estos análisis estadísticos comprende diferentes tipos de variables y distribuciones de los datos (Barceló 2009). La prueba estadística de chi-cuadrado

\begin{tabular}{lcccccccccc}
\hline & \multicolumn{1}{c}{ Femeninos } & \multicolumn{7}{c}{ Masculinos } \\
& n & Min & Max & B & Ds & n & Min & Max & B & Ds \\
\hline Húmero_LM & 7 & 278 & 304 & 291,93 & 10,36 & 25 & 298 & 346 & 326,2 & 13,4 \\
Húmero_EP & 7 & 40,11 & 43 & 41,45 & 1,03 & 25 & 41,33 & 51,74 & 47,67 & 3,18 \\
Húmero_ED & 7 & 52,05 & 57,73 & 55,24 & 2,01 & 25 & 50,07 & 70 & 63,7 & 4,08 \\
Valor-z Húmeros & 7 & & & $-1,32$ & 0,31 & 25 & & & 0,37 & 0,56 \\
Fémur_LM & 6 & 393,5 & 454 & 424,75 & 24,21 & 24 & 419,5 & 491 & 463,77 & 19 \\
Fémur_EP & 6 & 40,36 & 43,14 & 42,08 & 1,24 & 24 & 41,3 & 51,05 & 47,22 & 2,49 \\
Fémur_ED & 6 & 67 & 73,5 & 70 & 2,47 & 24 & 73 & 89 & 80,65 & 4,32 \\
Valor-z Fémur & 6 & & & $-1,34$ & 0,43 & 24 & & & 0,33 & 0,61 \\
Radio_LM & 9 & 211 & 249 & 233,33 & 12,78 & 18 & 230 & 273,5 & 259,75 & 12,3 \\
Radio_EP & 9 & 19,2 & 24,08 & 21,19 & 1,44 & 18 & 21,28 & 25,2 & 23,27 & 1,25 \\
Valor-z Radios & 9 & & & $-0,92$ & 0,71 & 18 & & & 0,46 & 0,63 \\
Cúbito_LM & 6 & 221 & 250 & 235,75 & 9,98 & 21 & 220 & 296,5 & 274,71 & 17,58 \\
Valor-z Cúbitos & 6 & & & $-1,32$ & 0,43 & 21 & & & 0,38 & 0,76 \\
Tibia_LM & 4 & 327 & 377 & 349 & 24,48 & 20 & 350 & 434 & 390,08 & 23,57 \\
Tibia_EP & 4 & 61 & 70 & 64,5 & 4,18 & 20 & 65,5 & 83 & 74,75 & 4,14 \\
Tibia_ED & 4 & 40 & 46 & 43,75 & 2,53 & 20 & 44 & 70 & 52,1 & 6,72 \\
Valor-z Tibias & 4 & & & $-1,25$ & 0,48 & 20 & & & 0,25 & 0,54 \\
\hline
\end{tabular}
evalúa la relación que existe entre dos o más niveles de un mismo factor cualitativo y otra variable cualitativa, con la ubicación de los datos en una tabla de contingencia. Este análisis compara los datos observados con unos datos teóricos, es decir aquellos

Tabla 1. Resultados de las mediciones anatómicas para la construcción de los valores-z según cada elemento anatómico. $\mathrm{LM}=$ longitud máxima; $\mathrm{EP}=$ epífisis próximal; $E D=$ epífisis distal. Se resaltan los valores-z. Todas las mediciones en $\mathrm{mm}$.

Table 1. Results of anatomic measurements for the construction of z-values according to each anatomical unit. $L M=$ Maximum bone length; $E P=$ Proximal epiphysis; $E D=$ Distal epiphysis. Z-values are highlighted. All measurements are in $\mathrm{mm}$. 
que se esperarían encontrar si no hubiese diferencias significativas. En cambio, la prueba U de Mann-Whitney utiliza los rangos de las mediciones para cada nivel del factor, cuyos resultados se basan en la comparación de sus rangos medios. Por otra parte, el estadístico $t$ de Student se basa en el cociente de la diferencia de las medias y la diferencia de las desviaciones típicas de cada nivel para la evaluación de diferencias significativas entre ellos. Por último, la correlación de rango de Spearman $\left(r_{s}\right)$ emplea diferencias de magnitud entre las mediciones, convertidas en rangos ordinales, de dos variables de igual o distinta naturaleza. Su resultado oscila entre -1 y +1 , siendo 0 la ausencia de relación. Para este último análisis, el sexo femenino fue codificado con el número 1, mientras que el sexo masculino se hizo con el número 2, con lo cual los coeficientes de correlación negativos señalarán mayores cambios entésicos a favor del sexo femenino; lo contrario para los coeficientes positivos.

Entre los factores degenerativos, endócrinos y genéticos que influyen en la manifestación de los cambios entésicos, se encuentra el tamaño corporal y la edad (e.g. Henderson y Nikita 2015). En primer lugar, se evaluó la presencia de asociaciones lineales significativas entre estas variables y los cambios entésicos a través de la correlación de rango de Spearman $(\alpha=0.050)$. Luego, se aplicaron dos correlaciones parciales de Spearman, cuyo objetivo es analizar la relación lineal entre dos variables mientras se controla/elimina el posible efecto de otra variable (Currell 2015). Entonces para esto se correlacionó el sexo de los individuos y los cambios entésicos, controlando, en uno de los análisis, el tamaño corporal y, en otro análisis, la edad. Se compararon estos resultados con los obtenidos en las otras pruebas.

Por último, se generaron dos MLG, uno con variable de respuesta de escala lineal y el otro con variable de respuesta logística ordinal, para cada una de las entesis $(\alpha=0.050)$. Estos modelos extienden la aplicación de regresiones lineales tradicionales a datos que no necesariamente cumplen con la condición de distribución normal (McCullagh y Nelder 1989). Es decir, los MLG pueden ser aplicados cuando una serie de variables dependientes, $y_{1}, y_{2} \ldots, y_{n}$ (también llamadas variables de respuesta) presentan cualquier tipo de distribución (normal, binomial, Poisson, ordinal, etc.) y son registradas como una función de $p$ variables explicativas (variables independientes), $X_{1}, X_{2^{\prime}} \ldots, X_{p^{\prime}}$ las cuales pueden ser continuas y/o categóricas. La expresión matemática del MLG es:

$$
\eta_{\mathrm{i}}=b_{0}+b_{1} X_{1}+b_{2} X_{2}+\ldots+b_{p} X_{p, i=1} 1,2, \ldots, \mathrm{n}
$$

donde la suma lineal ubicada a la derecha del signo igual se denomina predictor lineal e incluye los efectos de una o más variables explicativas; $b_{1} \ldots b_{p}$ representan los parámetros desconocidos que es necesario estimar; $\eta_{i}$ no representa $y_{n}$ sino a una transformación de los valores $y$ mediante una función de enlace. Es decir, que $\eta_{i}=g\left(\mu_{i}\right)$, donde $g$ es una función de enlace lineal monotónica de la media $\left(\mu_{\mathrm{i}}\right)$ de la función de distribución de la variable de respuesta $y$. Entonces, el MLG consiste en: 1- una distribución probabilística para la variable de respuesta $y, 2$ - un predictor lineal, y 3 - una función de enlace que relaciona el predictor lineal con la media de la función de distribución.

Ahora bien, dependiendo de la naturaleza de la variable de respuesta y (ordinal, escala, binaria) se desprenden una serie de funciones de distribución. En este trabajo, los cambios entésicos se analizaron de dos maneras: como variable de respuesta de escala lineal y como variable de respuesta logística ordinal. En el primer caso, la función de enlace es una función de identidad, donde $\eta_{i}=\mu_{i}$. En el segundo, cuando la variable de respuesta es ordinal, $\eta$ es una función log it acumulado, que se define como:

$$
\begin{gathered}
\eta_{i j}=\operatorname{logit}\left[P\left(y_{i} \leq j\right)\right] \\
=\log \left(\pi_{0}+\pi_{1}+\ldots+\pi_{j}\right) /\left(\pi_{j+1}+\ldots+\pi_{j}\right), j=0,1, \ldots, J-1
\end{gathered}
$$

donde $\pi$ es la probabilidad de que la variable $y_{i}$ tome el valor $j$.

Los MLG tienen la ventaja de permitir analizar y controlar los efectos de más de una variable al mismo tiempo. Es decir el modelo busca describir la influencia de una o más variables explicativas sobre una variable de respuesta. En este sentido, el MLG funciona al igual que las correlaciones parciales, explorando los efectos de cada predictor mientras controla los efectos de los otros predictores.

Las variables explicativas de este trabajo fueron la edad (dos categorías codificadas con el valor 1 para el rango etario 20-40; y con el valor 2 para >40 años), el sexo (dos categorías codificadas como 1 para el sexo femenino y 2 para el masculino) y los valores- $z$ de los elementos anatómicos (variable continua). La matriz de covarianza utilizada corresponde al estimador robusto, ya que provee de una buena estimación de la covarianza (Chrisletta y Spini 2004). El modelo elegido corresponde al de efectos principales con análisis tipo III. Este modelo evalúa la contribución (efecto) al modelo de cada variable explicativa mientras controla el efecto de las restantes.

Debido a que cuando se comparan más de dos variables aumenta la probabilidad de rechazar erróneamente la hipótesis nula, los valores $p$ fueron recalculados usando la corrección de Holm-Bonferroni para comparaciones múltiples' (Nikita 2017).

En todos los análisis la lateralidad derecha fue analizada

\footnotetext{
Los valores $p$ se ordenan de menor a mayor para luego ser multiplicados por el número de comparaciones $(\mathrm{N}, \mathrm{N}-1, \mathrm{~N}-2, \ldots ., 1$, respectivamente) (Nikita 2017).
} 
separada de la lateralidad izquierda ya que algunos individuos no contaban con ambas lateralidades.

Todos los análisis estadísticos se realizaron con programa IBM SPSS Statistics v. 19, con excepción de las correlaciones parciales de Spearman que se hicieron con R v. 3.1.2 ( $R$ Core Development Team 2014) a través de la función $p$ cor.test (Kim 2012).

\section{Resultados y Discusión}

La tabla 2 presenta una primera aproximación a la presencia de diferencias sexuales en los cambios entésicos a través de estadísticos univariados. Los análisis de frecuencias relativas muestran que ambos sexos manifiestan diferentes grados de robusticidad en casi todas las entesis. Sin embargo, los individuos femeninos presentan principalmente grados 1 y 2 , mientras que los masculinos tienen mayores frecuencias en el grado 3. Esta diferencia también se observa en el cálculo de las medias aritméticas de ambos sexos, donde los individuos masculinos presentan valores superiores a los femeninos, con excepción de la membrana interósea $y$ vasto medial derechos, dorsal ancho y membrana interósea izquierdos.

Si bien en la tabla 2 se observan diferencias entre los sexos, la tabla 3 muestra que no todas son estadísticamente significativas. De esto se desprende que los análisis

Tabla 2. Análisis estadísticos univariados y descriptivos para cada entesis, discriminadas por sexo y lateralidad.

Table 2. Univariate and descriptive statistical analyses for each enthesis, discriminated by sex and laterality.

\begin{tabular}{|c|c|c|c|c|c|c|c|c|c|c|c|c|c|}
\hline & & \multicolumn{6}{|c|}{ Femeninos } & \multicolumn{6}{|c|}{ Masculinos } \\
\hline & & \multirow{2}{*}{$n$} & \multicolumn{3}{|c|}{$\%$} & \multirow{2}{*}{ B } & \multirow{2}{*}{ ds } & \multirow{2}{*}{$n$} & \multicolumn{3}{|c|}{$\%$} & \multirow{2}{*}{ B } & \multirow{2}{*}{ ds } \\
\hline & & & Grado 1 & Grado 2 & Grado 3 & & & & Grado 1 & Grado 2 & Grado 3 & & \\
\hline & Lateralidad derecha & & & & & & & & & & & & \\
\hline 1 & Pectoral Mayor & 6 & 66,66 & 16,67 & 16,67 & 1,50 & 0,84 & 17 & 35,3 & 23,53 & 41,17 & 2,06 & 0,9 \\
\hline 2 & Dorsal Ancho & 6 & 100 & 0 & 0 & 1 & 0 & 16 & 75 & 18,75 & 6,25 & 1,31 & 0,6 \\
\hline 3 & Deltoides & 6 & 83,33 & 16,67 & 0 & 1,17 & 0,41 & 19 & 36,84 & 63,16 & 0,00 & 1,63 & 0,5 \\
\hline 4 & Braquiorradial & 6 & 83,33 & 16,67 & 0 & 1,17 & 0,41 & 16 & 75,00 & 25,00 & 0,00 & 1,25 & 0,45 \\
\hline 5 & Triceps & 5 & 100 & 0 & 0 & 1 & 0 & 15 & 73,33 & 26,67 & 0,00 & 1,27 & 0,46 \\
\hline 6 & Supinador & 5 & 60 & 40 & 0 & 1,4 & 0,55 & 16 & 56,25 & 37,50 & 6,25 & 1,5 & 0,63 \\
\hline 7 & Braquial anterior & 5 & 60 & 40 & 0 & 1,4 & 0,55 & 15 & 46,67 & 46,67 & 6,66 & 1,6 & 0,63 \\
\hline 8 & Biceps & 7 & 85,72 & 14,28 & 0 & 1,29 & 0,76 & 12 & 75 & 8,33 & 16,67 & 1,41 & 0,79 \\
\hline 9 & Pronador redondo & 6 & 100 & 0 & 0 & 1 & 0 & 13 & 92,30 & 7,70 & 0,00 & 1,08 & 0,28 \\
\hline 10 & Membrana interósea & 7 & 71,42 & 14,29 & 14,29 & 1,43 & 0,79 & 13 & 69,23 & 23,08 & 7,69 & 1,38 & 0,65 \\
\hline 11 & Glúteo Mayor & 6 & 83,33 & 16,67 & 0 & 1,17 & 0,41 & 20 & 55 & 25 & 20 & 1,65 & 0,81 \\
\hline 12 & Vasto medial & 6 & 50 & 50 & 0 & 1,5 & 0,55 & 20 & 95 & 5 & 0 & 1,05 & 0,22 \\
\hline 13 & Iliopsoas & 6 & 66,66 & 16,67 & 16,67 & 1,5 & 0,84 & 18 & 55,56 & 33,33 & 11,11 & 1,56 & 0,7 \\
\hline 14 & Cuádriceps & 4 & 75 & 25 & 0 & 1,25 & 0,5 & 15 & 60 & 33,33 & 6,67 & 1,47 & 0,64 \\
\hline \multirow[t]{2}{*}{15} & Sóleo & 4 & 75 & 25 & 0 & 1,25 & 0,5 & 15 & 73,33 & 26,67 & 0 & 1,27 & 0,46 \\
\hline & Lateralidad izquierda & & & & & & & & & & & & \\
\hline 1 & Pectoral Mayor & 3 & 66,66 & 33,34 & 0 & 1,33 & 0,58 & 20 & 55 & 20 & 25 & 1,7 & 0,86 \\
\hline 2 & Dorsal Ancho & 3 & 66,66 & 33,34 & 0 & 1,33 & 0,58 & 19 & 84,21 & 10,53 & 5,26 & 1,21 & 0,54 \\
\hline 3 & Deltoides & 3 & 66,66 & 33,34 & 0 & 1,33 & 0,58 & 19 & 42,1 & 47,37 & 10,53 & 1,68 & 0,67 \\
\hline 4 & Braquiorradial & 3 & 100 & 0 & 0 & 1 & 0 & 18 & 100 & 0 & 0 & 1 & 0 \\
\hline 5 & Tríceps & 4 & 100 & 0 & 0 & 1 & 0 & 12 & 83,33 & 0 & 16,67 & 1,33 & 0,78 \\
\hline 6 & Supinador & 4 & 100 & 0 & 0 & 1 & 0 & 12 & 33,33 & 58,33 & 8,34 & 1,75 & 0,62 \\
\hline 7 & Braquial anterior & 4 & 100 & 0 & 0 & 1 & 0 & 12 & 41,67 & 50 & 8,33 & 1,66 & 0,65 \\
\hline 8 & Biceps & 5 & 80 & 20 & 0 & 1,4 & 0,89 & 11 & 54,55 & 36,36 & 9,09 & 1,55 & 0,69 \\
\hline 9 & Pronador redondo & 4 & 100 & 0 & 0 & 1 & 0 & 12 & 75 & 25 & 0 & 1,25 & 0,45 \\
\hline 10 & Membrana interósea & 5 & 60 & 40 & 0 & 1,4 & 0,55 & 10 & 80 & 20 & 0 & 1,2 & 0,42 \\
\hline 11 & Glúteo Mayor & 5 & 80 & 0 & 20 & 1,4 & 0,89 & 21 & 38,1 & 28,57 & 33,33 & 1,95 & 0,86 \\
\hline 12 & Vasto medial & 5 & 100 & 0 & 0 & 1 & 0 & 20 & 90 & 10 & 0 & 1,1 & 0,31 \\
\hline 13 & lliopsoas & 5 & 60 & 20 & 20 & 1,6 & 0,89 & 19 & 42,1 & 47,37 & 10,53 & 1,68 & 0,67 \\
\hline 14 & Cuádriceps & 4 & 100 & 0 & 0 & 1 & 0 & 19 & 63,16 & 26,31 & 10,53 & 1,47 & 0,7 \\
\hline 15 & Sóleo & 4 & 75 & 25 & 0 & 1,25 & 0,5 & 18 & 83,33 & 16,67 & 0 & 1,17 & 0,38 \\
\hline
\end{tabular}

estadísticos univariados y descriptivos pueden usarse como primera aproximación para observar la forma en que se distribuyen los cambios entésicos en ambos sexos, pero no son concluyentes con respecto a la presencia de diferencias sexuales.

La correlación de rangos de Spearman es el análisis que muestra una mayor proporción de entesis con diferencias estadísticamente significativas entre ambos sexos (Tabla 3). Los coeficientes positivos en el deltoides derecho, supinador y braquial anterior izquierdo destacan que los individuos masculinos tienen mayores cambios entésicos que los femeninos en dichas entesis; mientras que el coeficiente negativo en el vasto medial derecho indica la presencia de mayores grados de robusticidad a favor de los individuos femeninos.

Llama la atención la cercanía de los valores $p$ de las pruebas $t$ de Student, $U$ de Mann-Whitney y correlación de rangos de Spearman, y su distancia con el análisis de Chi-cuadrado (Figura 2). Este último muestra diferencias estadísticamente significativas sólo en el vasto medial derecho. El estadístico $U$ de Mann-Whitney, si bien se aproxima a los resultados obtenidos con la correlación de Spearman, no muestra diferencias significativas en el deltoides derecho, como así tampoco junto a la $t$ de Student, lo hace para el braquial anterior izquierdo, aunque ambos presentan valores $p$ cercanos al establecido como límite de significación. Estas pequeñas discrepancias entre los estadísticos es producto de la naturaleza computacional de cada método. La falta de diferencias sexuales significativas con el empleo de la $U$ de Mann-Whitney indica que ambos sexos presentan los mismos rangos medios en dichas entesis; mientras que en la $t$ de Student señala una similitud en las medias aritméticas. A diferencia de estos dos estadísticos, el análisis de Chi-cuadrado no tiene en cuenta las medidas de tendencia central presentes en ambos sexos, sino que compara las frecuencias observadas con aquellas que se esperarían encontrar en caso de que no haya diferencias entre ambos sexos.

No es llamativo que la correlación de Spearman sea la prueba estadística que muestra diferencias significativas para una mayor cantidad de entesis, puesto que es el método más idóneo para analizar datos de naturaleza ordinal. De hecho, este método presenta una ventaja frente a los otros, dado que proporciona una medida de la intensidad de la asociación (coeficientes de correlación) con alguno de los dos niveles del factor, 


\begin{tabular}{|c|c|c|c|c|c|c|c|c|c|c|c|c|c|}
\hline & & \multicolumn{4}{|c|}{ Chi-cuadrado' } & \multicolumn{4}{|c|}{$\underline{\mathrm{U} \text { de Mann-Whitney }}$} & \multicolumn{2}{|c|}{ T-Student } & \multicolumn{2}{|c|}{ Correlación de Spearman } \\
\hline & & \multirow[t]{2}{*}{$n$} & \multirow{2}{*}{$\chi^{2}$} & \multirow{2}{*}{$g l$} & \multirow{2}{*}{$p$} & \multicolumn{2}{|c|}{ Suma de cuadrados } & \multirow{2}{*}{$u$} & \multirow{2}{*}{$p$} & \multirow{2}{*}{$t$} & \multirow{2}{*}{$p$} & \multirow{2}{*}{$r_{s}$} & \multirow{2}{*}{$p$} \\
\hline & & & & & & Fem. & Masc. & & & & & & \\
\hline \multicolumn{14}{|c|}{ Lateralidad derecha } \\
\hline 1 & Pectoral Mayor & 23 & 1,86 & 2 & 0,482 & 54,5 & 221,5 & 33,5 & 0,201 & $-1,33$ & 0,198 & 0,28 & 0,195 \\
\hline & Dorsal Ancho & 22 & 1,83 & 2 & 0,659 & 57 & 196 & 36 & 0,207 & $-1,25$ & 0,225 & 0,29 & 0,194 \\
\hline 3 & Deltoides & 25 & 3,94 & 1 & 0,073 & 51,5 & 273,5 & 30,5 & 0,056 & $-2,08$ & 0,049 & 0,4 & 0,049 \\
\hline & Braquiorradial & 22 & 0,17 & 1 & 1,00 & 65 & 188 & 44 & 0,723 & $-0,4$ & 0,695 & 0,09 & 0,695 \\
\hline 5 & Triceps & 20 & 1,66 & 1 & 0,530 & 42,5 & 167,5 & 27,5 & 0,232 & $-1,28$ & 0,217 & 0,29 & 0,217 \\
\hline 6 & Supinador & 21 & 0,32 & 2 & 1,00 & 52,5 & 178,5 & 37,5 & 0,850 & $-0,32$ & 0,755 & 0,05 & 0,820 \\
\hline 7 & Braquial anterior & 20 & 0,50 & 2 & 1,00 & 46,5 & 163,5 & 31,5 & 0,588 & $-0,63$ & 0,537 & 0,14 & 0,569 \\
\hline 8 & Bíceps & 19 & 0,66 & 2 & 1,00 & 66 & 124 & 38 & 0,677 & $-0,35$ & 0,728 & 0,11 & 0,648 \\
\hline 9 & Pronador redondo & 19 & 0,48 & 1 & 1 & 57 & 133 & 36 & 0,571 & $-0,67$ & 0,513 & 0,16 & 0,513 \\
\hline 10 & Membrana interósea & 20 & 0,37 & 2 & 1,00 & 73,5 & 136,5 & 45,5 & 0,961 & 0,13 & 0,895 & 0 & 1 \\
\hline 11 & Glúteo Mayor & 26 & 1,94 & 2 & 0,455 & 62 & 289 & 41 & 0,194 & $-1,39$ & 0,177 & 0,27 & 0,188 \\
\hline 12 & Vasto medial & 26 & 7,17 & 1 & 0,027 & 108 & 243 & 33 & 0,010 & 3,03 & 0,006 & $-0,53$ & 0,006 \\
\hline & Iliopsoas & 24 & 0,63 & 2 & 0,823 & 71 & 229 & 50 & 0,791 & $-0,16$ & 0,874 & 0,06 & 0,770 \\
\hline 14 & Cuádriceps & 19 & 0,44 & 2 & 1,00 & 35 & 155 & 25 & 0,595 & $-0,62$ & 0,541 & 0,14 & 0,570 \\
\hline 15 & Sóleo & 19 & 0,004 & 1 & 1,00 & 39,5 & 150,5 & 29,5 & 1 & $-0,06$ & 0,950 & 0,02 & 0,950 \\
\hline \multicolumn{14}{|c|}{ Lateralidad izquierda } \\
\hline 1 & Pectoral Mayor & 23 & 1,02 & 2 & 0,815 & 30 & 246 & 24 & 0,575 & $-0,7$ & 0,489 & 0,13 & 0,553 \\
\hline 2 & Dorsal Ancho & 22 & 1,24 & 2 & 0,469 & 39 & 214 & 24 & 0,569 & 0,37 & 0,718 & $-0,14$ & 0,534 \\
\hline 3 & Deltoides & 22 & 0,77 & 2 & 1,00 & 26,5 & 226,5 & 20,5 & 0,426 & $-0,85$ & 0,404 & 0,19 & 0,409 \\
\hline 4 & Braquiorradial & 21 & - & - & - & 33 & 198 & 27 & 0,959 & - & - & - & - \\
\hline 5 & Tríceps & 16 & 0,76 & 1 & 1,00 & 30 & 106 & 20 & 0,460 & $-0,84$ & 0,417 & 0,22 & 0,417 \\
\hline 6 & Supinador & 16 & 5,33 & 2 & 0,108 & 18 & 118 & 8 & 0,035 & $-2,36$ & 0,033 & 0,56 & 0,023 \\
\hline 7 & Braquial anterior & 16 & 4,14 & 2 & 0,130 & 20 & 116 & 10 & 0,062 & -2 & 0,065 & 0,5 & 0,049 \\
\hline 8 & Bíceps & 16 & 2,50 & 2 & 0,294 & 37,5 & 98,5 & 22,5 & 0,554 & $-0,36$ & 0,725 & 0,17 & 0,529 \\
\hline 9 & Pronador redondo & 16 & 0,12 & 1 & 0,528 & 28 & 108 & 18 & 0,325 & $-1,08$ & 0,298 & 0,28 & 0,298 \\
\hline 10 & Membrana interósea & 15 & 0,68 & 1 & 0,560 & 45 & 75 & 20 & 0,473 & 0,79 & 0,446 & $-0,21$ & 0,446 \\
\hline 11 & Glúteo Mayor & 26 & 3,19 & 2 & 0,209 & 48,5 & 302,5 & 33,5 & 0,195 & $-1,28$ & 0,214 & 0,27 & 0,188 \\
\hline 12 & Vasto medial & 25 & 0,54 & 1 & 1,00 & 60 & 265 & 45 & 0,516 & $-0,71$ & 0,482 & 0,15 & 0,482 \\
\hline 13 & Iliopsoas & 24 & 1,27 & 2 & 0,644 & 57,5 & 242,5 & 42,5 & 0,726 & $-0,23$ & 0,817 & 0,08 & 0,706 \\
\hline 14 & Cuádriceps & 23 & 2,11 & 2 & 0,533 & 34 & 242 & 24 & 0,176 & $-1,33$ & 0,196 & 0,3 & 0,165 \\
\hline 15 & Sóleo & 22 & 0,15 & 1 & 1,00 & 49 & 204 & 33 & 0,750 & 0,37 & 0,712 & $-0,08$ & 0,712 \\
\hline
\end{tabular}

- Mismo valor para todos los casos. No se puede realizar correlación.

Debido a que las frecuencias esperadas son menores a 5, se aplicó el estadístico Monte Carlo cuando los grados de libertad eran iguales a 2 y la prueba exacta de Fisher cuando los grados de libertad eran iguales a 1 (Barceló 2009).

Tabla 3. Resultados de los análisis estadísticos bivariados. Los resultados en negrita resaltan la presencia de diferencias estadísticamente significativas.

Table 3. Bivariate statistical results. Statistical significant differences are in bold. significativas entre ambos sexos en el dorsal ancho, deltoides y vasto medial derechos, y en el braquial anterior izquierdo. El vasto medial derecho es el único que tiene un coeficiente de correlación negativo, Io cual indica que los femeninos presentan mayores cambios entésicos que los masculinos en dicha entesis. En cambio, el dorsal ancho, deltoides derecho y braquial anterior izquierdo presentan mayores cambios entésicos entre los individuos masculinos.

En relación a la edad, no se detectaron correlaciones estadísticamente significativas con los cambios entésicos (Tabla 4). Sin embargo, al controlar la edad también detectaron diferencias estadísticamente significativas a favor de los individuos femeninos en el vasto medial derecho y, a favor de los masculinos, en el supinador y braquial anterior izquierdos.

Las correlaciones parciales coinciden con los resultados de los análisis bivariados en la presencia de diferencias sexuales en este caso el sexo de los individuos.

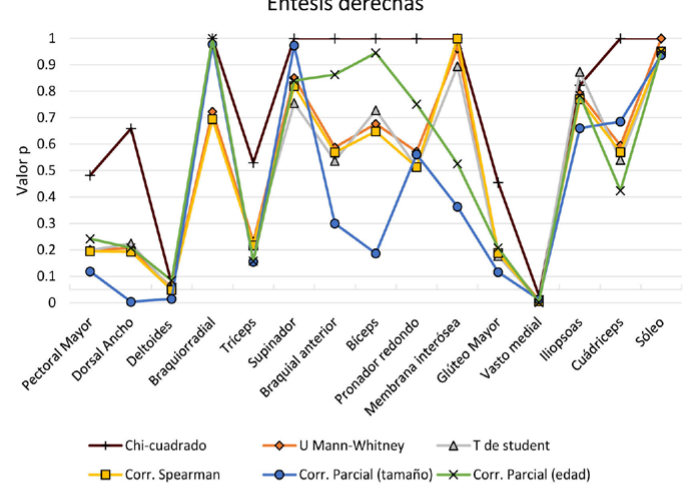

Entesis izquierdas

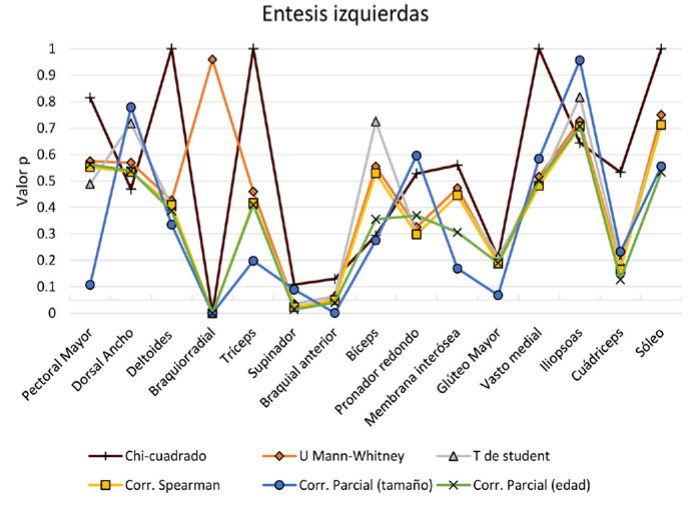

Relación entre cambios entésicos, tamaño corporal y edad

Dado que las diferencias sexuales en los cambios entésicos pueden estar influenciadas por el tamaño corporal y la edad, se realizaron correlaciones simples y parciales de Spearman. Con respecto a la influencia del tamaño corporal, se observó una asociación significativa positiva en el pronador redondo y bíceps izquierdo (Tabla 4), lo cual indica que los tamaños corporales más grandes presentan grados de cambios entésicos más elevados que los tamaños corporales más pequeños de la muestra. Al controlar la variable de tamaño, por medio de correlaciones parciales de Spearman, se detectaron diferencias
Figura 2. Comparación de los valores $p$ entre los análisis estadísticos bivariados para evaluar diferencias entre ambos sexos para cada entesis según su lateralidad. El símbolo alfa $(\alpha)$ señala el límite de significación (0.050).

Figure 2. P-value comparison among bivariate statistical analyses to test sexual differencesat each enthesis according to laterality. Alfa-symbol ( $\alpha)$ indicates the significance level (0.050). 


\begin{tabular}{|c|c|c|c|c|c|c|c|c|c|c|c|}
\hline & & \multicolumn{5}{|c|}{ Iamaño } & \multicolumn{5}{|c|}{ Edad } \\
\hline & & \multicolumn{3}{|c|}{$\underline{\text { Total }}$} & \multicolumn{2}{|c|}{$\underline{\text { Correlación parcial }}$} & \multicolumn{3}{|c|}{ Total } & \multicolumn{2}{|c|}{ Correlación parcial } \\
\hline & & $n_{\text {total }}$ & $r_{s}$ & $p$ & $r_{s}$ & $p$ & $n_{\text {total }}$ & $r_{s}$ & $p$ & $r_{s}$ & $p$ \\
\hline & Lateralidad derecha & & & & & & & & & & \\
\hline 1 & Pectoral Mayor & 23 & 0,09 & 0,695 & 0,32 & 0,119 & 23 & 0,15 & 0,486 & 0,25 & 0,242 \\
\hline 2 & Dorsal Ancho & 22 & $-0,08$ & 0,738 & 0,54 & 0,004 & 22 & 0,08 & 0,723 & 0,27 & 0,208 \\
\hline 3 & Deltoides & 25 & 0,12 & 0,561 & 0,46 & 0,015 & 25 & 0,29 & 0,156 & 0,34 & 0,084 \\
\hline 4 & Braquiorradial & 22 & 0,13 & 0,570 & $-0,006$ & 0,978 & 22 & 0,37 & 0,090 & 0 & 1 \\
\hline 5 & Triceps & 20 & 0,07 & 0,785 & 0,32 & 0,155 & 20 & $-0,10$ & 0,669 & 0,32 & 0,158 \\
\hline 6 & Supinador & 21 & 0,09 & 0,695 & $-0,007$ & 0,973 & 21 & 0,03 & 0,905 & 0,04 & 0,840 \\
\hline 7 & Braquial anterior & 20 & $-0,07$ & 0,754 & 0,24 & 0,300 & 20 & 0,42 & 0,066 & 0,04 & 0,863 \\
\hline 8 & Bíceps & 18 & 0,43 & 0,075 & $-0,32$ & 0,187 & 19 & 0,33 & 0,170 & $-0,01$ & 0,945 \\
\hline 9 & Pronador redondo & 18 & 0,40 & 0,102 & $-0,14$ & 0,561 & 19 & 0,31 & 0,199 & 0,08 & 0,751 \\
\hline 10 & Membrana interósea & 19 & 0,17 & 0,483 & $-0,22$ & 0,363 & 20 & 0,13 & 0,580 & $-0,15$ & 0,526 \\
\hline 11 & Glúteo Mayor & 26 & 0,04 & 0,842 & 0,31 & 0,116 & 26 & 0,34 & 0,094 & 0,25 & 0,208 \\
\hline 12 & Vasto medial & 26 & $-0,30$ & 0,139 & $-0,45$ & 0,013 & 26 & 0,15 & 0,466 & $-0,54$ & 0,001 \\
\hline 13 & Iliopsoas & 24 & $-0,01$ & 0,956 & 0,09 & 0,660 & 24 & 0,04 & 0,847 & 0,05 & 0,785 \\
\hline 14 & Cuádriceps & 19 & 0,10 & 0,695 & 0,10 & 0,685 & 19 & $-0,23$ & 0,340 & 0,19 & 0,424 \\
\hline \multirow[t]{2}{*}{15} & Sóleo & 19 & 0,04 & 0,859 & $-0,01$ & 0,937 & 19 & 0,15 & 0,543 & $-0,01$ & 0,950 \\
\hline & Lateralidad izquierda & & & & & & & & & & \\
\hline 1 & Pectoral Mayor & 23 & $-0,23$ & 0,282 & 0,33 & 0,107 & 23 & 0,19 & 0,382 & 0,12 & 0,562 \\
\hline 2 & Dorsal Ancho & 22 & $-0,15$ & 0,506 & $-0,06$ & 0,779 & 22 & $-0,16$ & 0,476 & $-0,13$ & 0,538 \\
\hline 3 & Deltoides & 22 & 0,02 & 0,929 & 0,21 & 0,335 & 22 & 0,38 & 0,085 & 0,19 & 0,387 \\
\hline 4 & Braquiorradial & 21 & - & - & - & - & 21 & - & - & - & - \\
\hline 5 & Triceps & 16 & $-0,02$ & 0,940 & 0,33 & 0,198 & 16 & 0 & 0,861 & 0,22 & 0,410 \\
\hline 6 & Supinador & 16 & 0,41 & 0,119 & 0,42 & 0,089 & 16 & 0,17 & 0,532 & 0,55 & 0,014 \\
\hline 7 & Braquial anterior & 16 & 0,04 & 0,875 & 0,67 & $<0.001$ & 16 & 0,06 & 0,819 & 0,49 & 0,039 \\
\hline 8 & Bíceps & 16 & 0,51 & 0,046 & $-0,28$ & 0,275 & 16 & $-0,2$ & 0,469 & 0,24 & 0,355 \\
\hline 9 & Pronador redondo & 16 & 0,57 & 0,020 & $-0,14$ & 0,596 & 16 & 0,37 & 0,156 & 0,24 & 0,369 \\
\hline 10 & Membrana interósea & 15 & 0,07 & 0,805 & $-0,36$ & 0,169 & 15 & 0,18 & 0,510 & $-0,28$ & 0,306 \\
\hline 11 & Glúteo Mayor & 26 & $-0,02$ & 0,923 & 0,35 & 0,068 & 26 & 0,34 & 0,092 & 0,26 & 0,190 \\
\hline 12 & Vasto medial & 25 & 0,08 & 0,698 & 0,11 & 0,584 & 25 & 0,22 & 0,288 & 0,14 & 0,501 \\
\hline 13 & Iliopsoas & 24 & 0,13 & 0,534 & $-0,01$ & 0,956 & 24 & $-0,01$ & 0,975 & 0,08 & 0,707 \\
\hline 14 & Cuádriceps & 23 & 0,16 & 0,459 & 0,25 & 0,232 & 23 & $-0,12$ & 0,588 & 0,32 & 0,127 \\
\hline 15 & Sóleo & 22 & $-0,28$ & 0,209 & 0,13 & 0,555 & 22 & 0,36 & 0,104 & $-0,14$ & 0,533 \\
\hline
\end{tabular}

Mismo valor para todos los casos. No se puede realizar correlación.

en el vasto medial derecho y braquial anterior izquierdo. De hecho, todas las pruebas estadísticas señalan la presencia de diferencias sexuales en el vasto medial derecho. En el deltoides derecho y supinador izquierdo también fueron detectadas diferencias entre ambos sexos con los análisis bivariados y las correlaciones parciales. Sin embargo, en la primera entesis, las diferencias se encontraron solamente cuando se controló el tamaño corporal y no así cuando se controló la edad; lo contrario ocurre con el supinador. Esto nos advierte que, si bien los análisis bivariados arrojaron diferencias entre ambos sexos en ambas entesis, es posible que estas diferencias estén influenciadas por factores etarios, en el caso del deltoides derecho, y por el tamaño corporal, en el caso del supinador izquierdo ya que las diferencias sexuales no fueron detectadas por ambas correlaciones parciales (controlando el tamaño y controlando la edad).

Estos análisis señalan la importancia de tener en cuenta el tamaño corporal y la edad de los individuos al analizar diferencias sexuales en los cambios entésicos. Sin embargo, las correlaciones parciales no permiten la inclusión de más de una variable de control en el análisis. Es por esta razón que es importante analizar las manifestaciones óseas que tienen una etiología multifactorial con estadísticos que permitan controlar más de una variable. Este es el caso de los modelos lineales generalizados.
Tabla 4. Resultados de las correlaciones simples y parciales de Spearman. Los resultados en negrita resaltan la presencia de asociaciones estadísticamente significativas.

Table 4. Results of Spearman's simple and partial correlations. Statistical significant differences are in bold.

Los resultados de ambos MLG muestran que el factor que más influye en los cambios entésicos es el sexo de los individuos, teniendo en cuenta solamente los valores significativos luego de la corrección Holm-Bonferroni. A esta variable le sigue, en orden de importancia, la edad de los individuos y el tamaño corporal en el caso del MLG ordinal, o a la inversa en el caso del MLG de escala (Tabla 5).

Por otro lado, en la tabla 5 también se puede observar que la cantidad de entesis que muestran diferencias significativas entre las dos categorías etarias, sexos y tamaños corporales es mayor que la detectada con los análisis estadísticos bivariados. A su vez, los MLG mostraron asociaciones entre los cambios entésicos y la edad de los individuos, correlación que estaba ausente en los análisis bivariados (Figura 3).

Al comparar ambos MLG (ordinal y escala) observamos algunas diferencias entre ellos. En primer lugar, el MLG ordinal presenta valores del criterio de información de Akaike corregido ( $\mathrm{AIC}_{\mathrm{C}}$ ) menores al MLG de escala (con excepción del vasto medial en ambas lateralidades), lo cual indica que la calidad del modelo ordinal es mejor (Hurvich y Tsai 1989; Sugiura 1978). En segundo lugar, si bien algunas entesis mostraron relaciones significativas con las variables explicativas, el MLG ordinal presenta mayores resultados significativos que el MLG de escala. Esto posiblemente se deba a la naturaleza computacional de cada uno de ellos. Como se mencionó en el apartado metodológico, cada uno de los modelos utiliza diferentes funciones de enlace. Sin embargo, algunos resultados fueron significativos en ambos modelos (ver valores subrayados en la tabla 5).

Los valores- $B$ de la tabla 5 son los valores de los párametros de los modelos. Ellos nos indican la dirección que asume la variable en cuestión. En ambos modelos y en el caso de la edad, varias entesis con resultados significativos aún luego de la corrección Holm-Bonferroni, presentan mayores cambios entésicos entre los individuos mayores a 40 años (parámetros con signo negativo). La presencia de cambios entésicos más avanzados entre los individuos más adultos de la muestra ya fue detectada 


\begin{tabular}{|c|c|c|c|c|c|c|c|c|c|c|c|c|c|c|c|}
\hline & & \multicolumn{7}{|c|}{ MLG ordinal } & \multicolumn{7}{|c|}{ MLG escala } \\
\hline & & \multirow{2}{*}{$\mathrm{AlC}_{\mathrm{C}}$} & \multicolumn{2}{|c|}{$\underline{\text { Edad }}$} & \multicolumn{2}{|c|}{$\underline{\operatorname{sexo}}$} & \multicolumn{2}{|c|}{ Tamaño } & \multirow{2}{*}{$\mathrm{AlC}_{\mathrm{C}}$} & \multicolumn{2}{|c|}{$\underline{\text { Edad }}$} & \multicolumn{2}{|c|}{$\underline{\operatorname{Sexo}}$} & \multicolumn{2}{|c|}{ Tamaño } \\
\hline & & & $B$ & $p$ & $B$ & $p$ & $B$ & $p$ & & $B$ & $p$ & $B$ & $p$ & $B$ & $p$ \\
\hline \multicolumn{16}{|c|}{ Entesis derechas } \\
\hline 1 & Pectoral Mayor & 59,26 & $-0,275$ & 0,802 & -2.656 & 0,093 & $-0,838$ & 0,248 & 69,36 & $-0,164$ & 0,687 & -1.320 & 0,029 & $-0,454$ & 0,144 \\
\hline 2 & Dorsal Ancho & 32,65 & $-0,241$ & 0,813 & -23.586 & $\leq 0.001^{*}$ & -1.866 & $\underline{0.016^{*}}$ & 41,23 & $-0,053$ & 0,714 & -1.049 & $\underline{0.005^{*}}$ & $-0,427$ & $\underline{0.011^{*}}$ \\
\hline 3 & Deltoides & 35,24 & -1.507 & 0,271 & -7.008 & $0.014^{*}$ & -2.592 & 0,068 & 40,49 & 1.314 & 0,252 & 20.657 & $<0.001^{*}$ & 6.682 & $0.010^{*}$ \\
\hline 4 & Braquiorradial & 29,42 & -29.956 & $\leq 0.001^{*}$ & 0,328 & 0,901 & 0,180 & 0,880 & 34,63 & $\underline{-0,334}$ & $\underline{0.009^{*}}$ & 0,050 & 0,891 & 0,029 & 0,878 \\
\hline 5 & Tríceps & 27,22 & 1.029 & 0,404 & -21.585 & $<0.001^{*}$ & $-0,310$ & 0,616 & 31,77 & 0,150 & 0,415 & $-0,408$ & 0,139 & $-0,053$ & 0,650 \\
\hline 6 & Supinador & 46,91 & $-0,110$ & 0,918 & 0,832 & 0,619 & 0,531 & 0,369 & 50,74 & 0,059 & 0,853 & 0,078 & 0,855 & 0,102 & 0,420 \\
\hline 7 & Braquial anterior & 43,32 & -1.785 & 0,074 & $-0,532$ & 0,810 & $-0,127$ & 0,901 & 46,04 & $-0,485$ & 0,038 & $-0,099$ & 0,840 & $-0,015$ & 0,948 \\
\hline 8 & Bíceps & 30,06 & -4.361 & $0.001^{*}$ & 7.041 & $0.027^{*}$ & 3.694 & $\underline{0.012^{*}}$ & 48,83 & $-0,613$ & 0,042 & 1.035 & 0,075 & $\underline{0,585}$ & $\underline{0,022}$ \\
\hline 9 & Pronador redondo & -* & $-^{*}$ & -* & $-^{*}$ & $-^{*}$ & -* & $-^{*}$ & 9,73 & $-0,145$ & 0,249 & 0,088 & 0,307 & 0,087 & 0,243 \\
\hline 10 & Membrana interósea & 40,51 & -1.776 & 0,068 & 2.738 & 0,156 & 1.023 & 0,212 & 50,71 & $-0,371$ & 0,155 & 0,576 & 0,197 & 0,240 & 0,196 \\
\hline 11 & Glúteo Mayor & 56,05 & -1.556 & 0,137 & -1.471 & 0,295 & 0,025 & 0,968 & 67,13 & $-0,443$ & 0,118 & $-0,372$ & 0,391 & 0,040 & 0,865 \\
\hline 12 & Vasto medial & 24,36 & -1.896 & 0,399 & 4.815 & 0,017 & 0,801 & 0,230 & 23,53 & $-0,167$ & 0,194 & 0,581 & $0.003^{*}$ & 0,068 & 0,279 \\
\hline 13 & lliopsoas & 57,99 & $-0,093$ & 0,915 & $-0,464$ & 0,755 & $-0,106$ & 0,853 & 64,63 & $-0,056$ & 0,854 & $-0,093$ & 0,857 & $-0,027$ & 0,895 \\
\hline 14 & Cuádriceps & 42,89 & 1.530 & 0,193 & 0,080 & 0,966 & 0,845 & 0,332 & 45,19 & 0,471 & 0,143 & 0,023 & 0,953 & 0,233 & 0,252 \\
\hline 15 & Sóleo & 32,16 & $-0,716$ & 0,617 & 0,695 & 0,652 & 0,421 & 0,625 & 36,78 & $-0,125$ & 0,587 & 0,132 & 0,646 & 0,079 & 0,619 \\
\hline \multicolumn{16}{|c|}{ Entesís izquierdas } \\
\hline 1 & Pectoral Mayor & 54,60 & $-0,885$ & 0,364 & -3.615 & 0,083 & -1.571 & 0,065 & 63,89 & $-0,357$ & 0,220 & -1.691 & 0,022 & $-0,702$ & 0,018 \\
\hline 2 & Dorsal Ancho & 38,16 & 0,841 & 0,455 & 0,573 & 0,837 & $-0,178$ & 0,869 & 46,73 & $-0,081$ & 0,716 & $-0,004$ & 0,995 & $-0,066$ & 0,731 \\
\hline 3 & Deltoides & 50,35 & -1.804 & 0,083 & -2.328 & 0,276 & $-0,586$ & 0,518 & 51,77 & $-0,523$ & 0,036 & $-0,795$ & 0,203 & $-0,239$ & 0,375 \\
\hline 4 & Braquiorradial & - & - & - & - & - & - & - & - & - & - & - & - & - & - \\
\hline 5 & Triceps & 20,62 & 0,356 & 0,824 & -22.006 & $<0.001^{*}$ & $-0,836$ & 0,285 & 40,94 & 0,037 & 0,914 & $-0,668$ & 0,227 & $-0,187$ & 0,414 \\
\hline 6 & Supinador & 35,27 & $-0,846$ & 0,414 & -22.608 & $\leq 0.001^{*}$ & $-0,443$ & 0,566 & 39,39 & $-0,222$ & 0,329 & $-0,943$ & $\leq 0.001^{*}$ & $-0,120$ & 0,463 \\
\hline 7 & Braquial anterior & 32,82 & $-0,654$ & 0,523 & -25.570 & $\leq 0.001^{*}$ & -2.146 & $\underline{0.021^{*}}$ & 37,36 & $-0,210$ & 0,385 & -1.515 & $\leq 0.001^{*}$ & $-0,489$ & $\underline{0.006^{*}}$ \\
\hline 8 & Bíceps & 35,89 & 1.603 & 0,131 & 1.873 & 0,468 & 3.341 & 0,020 & 42,89 & 0,311 & 0,183 & 0,522 & 0,279 & 0,581 & $0.008^{\star}$ \\
\hline 9 & Pronador redondo & 19,14 & -19.677 & $<0.001^{*}$ & -15.412 & $<0.001^{*}$ & 2.943 & 0,168 & 25,28 & $-0,217$ & 0,060 & 0,082 & 0,537 & 0,204 & 0,044 \\
\hline 10 & Membrana interósea & 22,99 & -3.952 & 0,074 & 6.438 & 0,075 & 2.987 & 0,061 & 29,24 & $-0,282$ & 0,091 & 0,651 & 0,023 & 0,295 & 0,024 \\
\hline 11 & Glúteo Mayor & 62,84 & -1.414 & 0,088 & -1.603 & 0,357 & 0,028 & 0,965 & 74,32 & $-0,597$ & 0,057 & $-0,476$ & 0,429 & 0,021 & 0,939 \\
\hline 12 & Vasto medial & 20,80 & -20.682 & $<0.001^{*}$ & -18.678 & $<0.001^{*}$ & 0,807 & 0,261 & 16,78 & $-0,136$ & 0,132 & $-0,002$ & 0,984 & 0,054 & 0,327 \\
\hline 13 & Iliopsoas & 59,12 & $-0,110$ & 0,886 & 1.197 & 0,514 & 0,933 & 0,272 & 62,37 & $-0,059$ & 0,826 & 0,429 & 0,470 & 0,290 & 0,261 \\
\hline 14 & Cuádriceps & 45,56 & 0,929 & 0,356 & -19.861 & $<0.001^{*}$ & 0,771 & 0,312 & 55 & 0,263 & 0,347 & $-0,182$ & 0,446 & 0,225 & 0,222 \\
\hline 15 & Sóleo & 24,38 & $-21,54$ & $\leq 0.001^{*}$ & $-0,949$ & 0,630 & -1.736 & 0,117 & 28,58 & $-0,331$ & $\underline{0.012^{*}}$ & $-0,184$ & 0,547 & $-0,215$ & 0,141 \\
\hline
\end{tabular}

Tabla 5. Resultados del modelo lineal generalizado para cada entesis. Los resultados en negrita resaltan la presencia de diferencias estadísticamente significativas.

Table 5. Results of generalized linear models at each enthesis. Statistical significant differences are in bold.

para otras poblaciones pasadas (e.g. Alves Cardoso y Henderson 2010; Mariotti et al. 2004). Esto podría deberse a cambios degenerativos que acontecen en edades avanzadas, ya sea en las células óseas, las cuales provocan una apariencia más rugosa en las entesis (Weiss et al. 2012), o bien en los tejidos conectivos, lo cual aumenta la exposición al estrés mecánico (Jurmain et al. 2012). Por otro lado, también se ha propuesto que las diferencias etarias en los cambios entésicos podrían estar relacionadas con una acumulación de estrés mecánico durante la vida del individuo (Molnar 2010; Robb 1998).

Con respecto a las diferencias entre ambos sexos, los MLG, coinciden con las resultados de las correlaciones parciales de Spearman, y muestran diferencias significativas en seis entesis más en el caso del MLG ordinal (tríceps y bíceps derechos, tríceps, pronador redondo, vasto medial y cuádriceps izquierdos) (Tabla 5; Figura 3). No sólo las correlaciones parciales de Spearman no detectaron las diferencias significativas entre ambos sexos en estas últimas seis entesis, sino que tampoco habían sido detectadas por los análisis bivariados. Los MLG, al controlar la edad y el tamaño corporal al mismo tiempo, fueron capaces de encontrar mayores diferencias entre ambos sexos.

Al analizar los valores de los parámetros para la variable del sexo de los individuos, observamos que los masculinos presentan mayores grados de robusticidad que los femeninos en la mayoría de las entesis con valores $p$ significativos (parámetros con signo negativo), con excepción del bíceps derecho en el caso del MLG ordinal y del detoides y vasto medial derecho para el MLG de escala (parámetros con signo positivo). Si bien estas diferencias sexuales pueden ser producto de la acción de andrógenos (Notelovitz 2002), es llamativo que algunas entesis presenten mayores cambios entésicos entre los individuos femeninos sólo en los miembros superiores. Por el contrario, las entesis de los miembros inferiores, tal como puede observarse en la tabla 5, guardan relación únicamente con el sexo masculino. Por lo tanto, es posible que estas diferencias estén influenciadas no sólo por el 
A)
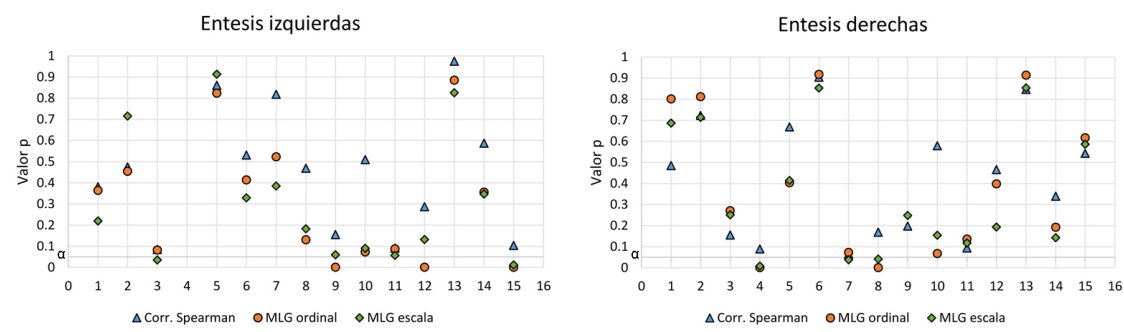

B)
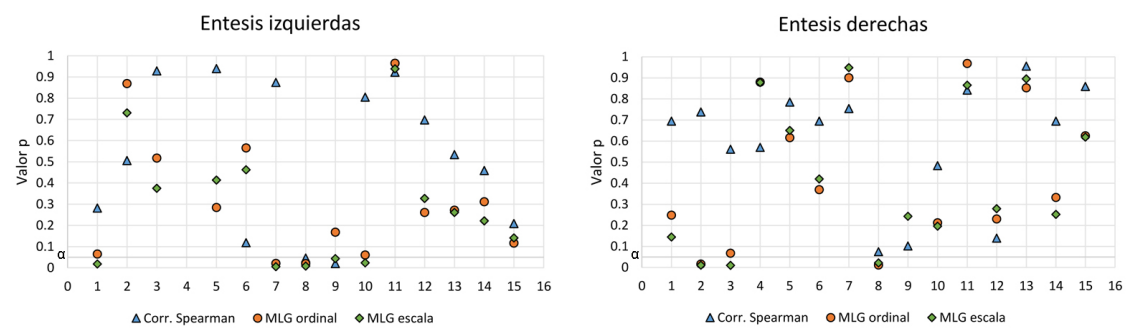

C)
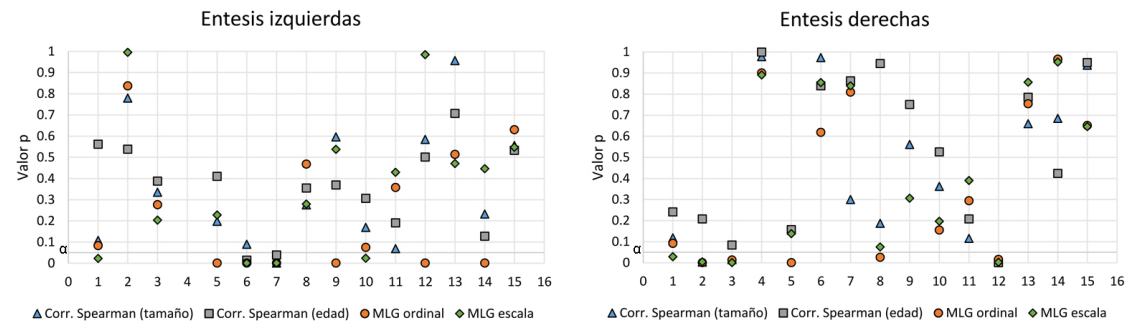

Figura 3. Comparación de los valores $p$ entre las correlaciones simples y parciales de Spearman y del modelo lineal generalizado para cada entesis (1-15) según su lateralidad con respecto a: A) edad; B) tamaño corporal; C) sexo. El símbolo $\alpha$ señala el límite de significación (0.05).

Figure 3. P-value comparison among spearman simple and partial correlations and generalized linear models at each enthesis (1-15) according to laterality. A) Age; B) Body size; C) Sex. Alfa-symbol $(\alpha)$ indicates the significance limit (0.05). efecto anabólico de las hormonas esteroides sino también por las actividades realizadas por ambos sexos. Mientras que las diferencias observadas en los miembros superiores podrían deberse a una amplia variedad de actividades, las correspondientes a los miembros inferiores podrían ser el resultado de, por un lado, diferencias hormonales entre ambos sexos, pero también, por otro lado, podrían indicar que los individuos masculinos realizaban actividades ambulatorias de mayor intensidad que los femeninos.

Finalmente, los MLG muestran asociaciones significativas entre los cambios entésicos y el tamaño corporal en una mayor cantidad de entesis que aquellas registradas con las correlaciones de Spearman (Tabla 5; Figura 3). Sin embargo, no existe una única dirección en la asociación. En ambos modelos, el bíceps derecho muestra mayores cambios entésicos entre los tamaños corporales más grandes (parámetros positivos). Lo mismo sucede si consideramos los resultados del MLG de escala para el deltoides derecho y bíceps izquierdo. Este tipo de asociación también fue hallada por otros investigadores (e.g.Weiss 2003; Zumwalt 2006), cuya relación fue atribuida al esfuerzo físico que requieren los tamaños corporales grandes para moverse (Krantz 1981). Por contrario a estos resultados, el dorsal ancho derecho y el braquial anterior izquierdo en ambos modelos presentan una dirección inversa (parámetros negativos). Es decir que los mayores cambios entésicos se observan entre los tamaños corporales más pequeños de la muestra. Aun no se han encontrado relaciones entre el tamaño de las superficies de las entesis y el tamaño corporal (Henderson 2013a) como para atribuir estos resultados a una mayor presión mecánica sobre entesis pequeñas. Por lo tanto, es posible que esta relación inversa entre tamaño corporal y cambios entésicos sea producto del tamaño de la muestra o consecuencia del estrés físico. Ambas opciones deben analizarse en muestras con una mayor cantidad de individuos.

Las discrepancias entre los resultados de las correlaciones de Spearman (simples y parciales) y los MLG puede deberse a que los índices de significación de las correlaciones se refieren a sus coeficientes $r$, mientras que aquellos de los MLG remiten a los coeficientes del modelo $\left(b_{1}, b_{2}, \ldots, b_{n}\right)$ (Nikita 2014). A su vez, los valores $p$ de estos coeficientes se obtienen luego de controlar las otras variables, a diferencia de las correlaciones de rango simples de Spearman que no incluyen variables de control o de la correlación parcial de Spearman que sólo controla una variable a la vez.

Es importante mencionar una de las limitaciones que 
tiene este trabajo con respecto al tamaño muestral. Los resultados expuestos deben continuar poniéndose a prueba en muestras mayores con individuos completos que permitan estimar la edad y el tamaño corporal. A su vez, si bien no es parte del objetivo de este trabajo, alguna de las diferencias observadas entre los individuos con respecto a los cambios entésicos pueden deberse a diferencias cronológicas o a una variabilidad entre los sitios arqueológicos. Al día de la fecha, la cantidad de fechados radiocarbónicos no nos permite evaluar la presencia de diferencias temporales a nivel del individuo.

\section{Conclusiones}

En este trabajo se han aplicado y discutido una serie de estadísticos comúnmente utilizados para evaluar la presencia de diferencias sexuales en los cambios entésicos a partir de una muestra proveniente del humedal del Paraná inferior. Si bien todos los análisis muestran que ambos sexos difieren en los cambios entésicos, su etiología multifactorial requiere de pruebas estadísticas que puedan incorporar al análisis al menos el control de una covariable. De esta manera se puede evaluar la influencia de otros factores que afectan a las entesis, como ser el tamaño corporal y la edad de los individuos.

La aplicación de modelos lineales generalizados en estudios de variables óseas con etiología multifactorial ha sido impulsada en los últimos años (Henderson y Nikita 2015; Nikita 2014). Este método presenta la ventaja de poder controlar el efecto de varias variables explicativas sobre la variable dependiente al mismo tiempo. De esta forma, proporciona resultados más confiables que aquellos derivados de sucesivas comparaciones a través de métodos bivariados, como ser las correlaciones parciales. Por supuesto debe considerarse que cada uno de los métodos expuestos posee diferentes propiedades computacionales, complejidad estadística, etc. que pueden, en ciertas ocasiones, ser más viables de aplicar que otros.

Ciudad Autónoma de Buenos Aires, 13 de Abril de 2017

\section{Agradecimientos}

A los dos evaluadores anónimos de este artículo que contribuyeron con sus comentarios y correcciones. A los responsables de la División de Antropología Biológica del Museo de La Plata (Dr. Mariano del Papa y Andrés Di Bastiano) por facilitarme el acceso a las colecciones arqueológicas de la institución. A los Dres. Marcelo Cardillo y Efthymia Nikita por su ayuda con uno de los análisis estadísticos. A los Dres. Daniel Loponte, Alejandro Acosta y Gustavo Barrientos por ser mis guías y fuentes de consulta durante las investigaciones. Esta investigación fue realizada con una beca de investigación doctoral y postdoctoral del CONICET. Todo lo vertido es única responsabilidad de la autora.

\section{Bibliografía}

Acosta, A., B. Mazza. 2016. Restos óseos humanos y faunísticos: su relación en el espacio mortuorio en contextos de cazadores-recolectores del humedal del Paraná inferior. Pesquisas Antropologia, 72: 185-207.

Aguado, I., M. Porta Serra, M. Miralles, F. García Benavides y F. Bolúmar. 1990. La cuantificación de variabilidad en las observaciones clínicas. Medicina Clínica, 95: 424-429.

Alves Cardoso, F., C. Henderson. 2010. Enthesopathy formation in the humerus: Data from known age-at-death and known occupation skeletal collections. American Journal of Physical Anthropology, 141: 550-560.

Auerbach, B., C. Ruff. 2010. Stature estimation formulae for indigenous North American populations. American Journal of Physical Anthropology, 141: 190-207.

Barceló, J. 2009. Arqueología y estadística. Introducción al estudio de la variabilidad de las evidencias arqueológicas. Universitat Autònoma de Barcelona Servei de Publicacions, España.

Brooks, S.T., J.M. Suchey. 1990. Skeletal age determination based on the os pubis: a comparison of the AcsádiNemeskéri and Suchey-Brooks methods. Human Evolution, 5: 227-238.

Buikstra, J., D. Ubelaker. 1994. Standards for Data Collection from Human Skeletal Remains. Arkansas Archeological Survey, Arkansas.

Caggiano, M.A., O.B. Flores, M.G. Méndez y S.A. Salceda. 1978. Nuevos aportes para el conocimiento antropológico del Delta del Paraná. Relaciones de la Sociedad Argentina de Antropología, XII: 155-174.

Currell, G. 2015. Scientific data analysis. Oxford University Press, Inglaterra.

Chrisletta, P., D. Spini. 2004. An introduction to generalized estimating equations and an application to assess selectivity effects in a longitudinal study on very old individuals. Journal of Educational and Behavioral Statistics, 29: 421-437.

Hagaman, K. 2009. "Activity-induced musculoskeletal stress marker analysis of the Windover population". Florida State University, College of Arts and Sciences, Department of Anthropology, Estados Unidos, 130 p.Degree of Master of Arts. Electronic Theses, Treatises and Dissertations.

Henderson, C. 2013a. Technical note: Quantifying size and shape of entheses. Antropological Science, 121 (1): 63-73. 
Henderson, C. 2013b. Do diseases cause entheseal changes at fibrous entheses? International Journal of Paleopathology, 3: 64-69.

Henderson, C., E. Nikita. 2015. Accounting for multiple effects and the problem of small sample sizes in osteology: A case study focussing on entheseal changes. Archaeological Antropological Science, DOI 10.1007/ s12520-015-0256-1.

Hurvich, C. M., C. L. Tsai. 1989. Regression and time series model selection in small samples. Biometrika, 76: 297-307

Jurmain, R., F. Alves Cardoso y C. Henderson. 2012. Bioarchaeology's holy grail: the reconstruction of activity. Grauer, A.L. (ed.), A Companion to Paleopathology, pp. 531-552. Blackwell, New York.

Kim, S. 2012. ppcor: Partial and Semi-partial (Part) correlation. R package version 1.0. http://CRAN.R-project. org/package=ppcor.

Kramer, M., A. Feinstein. 1981. Clinical biostatistics. LIV. The biostatistics of concordance. Clinical Pharmacology \& Therapeutics, 29 (1): 111-123.

Krantz, G.S. 1981. The process of human evolution. Schenkman Publishing Company Inc., Cambridge.

Loponte, D. 2008. Arqueología del Humedal del Paraná Inferior. Acosta, A., D. Loponte (eds.), Arqueología de la Cuenca del Plata. Serie Monográfica 1. Ediciones del Riel, Buenos Aires.

Loponte, D., A. Acosta. 2011. Informe de avance de la investigación en el sitio El Cazador sitio 3. Secretaría de Cultura de la Nación, Instituto Nacional de Antropología y Pensamiento Latinoamericano.

Loponte, D., A. Acosta y P. Tchilingurian. 2015. El sitio arqueológico Escuela 31. Revista del Museo de la Plata, Arqueología, 14 (89): 27R.

Lothrop, S. 1932. Indians of the Parana Delta, Argentina. Annals of the New York Academy of Scienses, 33: 77-232.

Lovejoy, O., R. Meindl, T. Pryzbeck y R. Mensforth. 1985. Chronological metamorphosis of the auricular surface of the ilium: A new method for the determination of adult skeletal age at death. American Journal of Physical Anthropology, 68: 15-28.

Mariotti, V., F. Facchini y M.G. Belcastro. 2004. Enthesopathies - Proposal of standardized scoring method and applications. Collegium Antropologicum, 28: 145-159.

Mariotti, V., F. Facchini y M. Giovanna Belcastro. 2007.
The Study of Entheses: Proposal of a Standardised Scoring Method for Twenty-Three Entheses of the Postcranial Skeleton. Collegium Antropologicum, 31: 291-313.

Mazza, B. 2010. Cerro Lutz: Aproximaciones al estudio de las prácticas mortuorias de las sociedades cazadorasrecolectoras del humedal del Paraná inferior. Revista La Zaranda de Ideas, Revista de Jóvenes Investigadores en Arqueología, 6: 91-116.

Mazza, B. 2015. Colecciones antiguas, datos nuevos: Primeros resultados del análisis de las colecciones bioarqueológicas del humedal del Paraná inferior. Revista del Museo de Antropología, 8 (1): 133-146.

McCullagh, P., J.A. Nelder. 1989. Generalized linear models. 2da edición. Chapman and Hall, Londres.

Meindl, R., O. Lovejoy. 1985. Ectocranial suture closure: A revised method for the determination of skeletal age at death based on the lateral-anterior sutures. Journal of Physical Anthropology, 68: 57-66.

Molnar, P. 2010. Patterns of physical activity and material culture on Gotland, Sweden, during the Middel Neolithic. International Journal of Osteoarchaeology, 20: 1-14.

Nikita, E. 2014. The use of generalized linear models and generalized estimating equations in bioarchaeological studies. American Journal of Physical Anthropology, 153: 473-483.

Nikita, E. 2017. Osteoarchaeology. A guide to the macroscopic study of human skeletal remains. Academic Press, Amsterdam.

Niinimäki, J. 2012. The relationship betwwen musculoskeletal stress markers and biomechanical propierties of the humeral diaphysis. American Journal of Physical Anthropology, 147: 618-628.

Notelovitz, M. 2002. Androgen effects on bone and muscle. Fertility and Sterility, 77 (4): S34-41.

Peterson, J. 1998. The Natufian hunting conundrum: spears, atlats, or bows? Musculoskeletal and armature evidence. International Journal of Osteoarchaeology, 8: 378-389.

Phenice, T. 1969. A new developed visual method of sexing in the os pubis. American Journal of Physical Anthropology, 30: 297-301.

R Core Team. 2014. R: A language and environment for statistical computing. R Foundation for Statistical Computing, Vienna, Austria. URL http://www.R-project.org/.

Raxter, M., B. Auerbach y C. Ruff. 2006. Revision of the 
fully technique for estimating statures. American Journal of Physical Anthropology, 130: 374-384.

Resnick, D., G. Niwayama. 1983. Enthesis and enthesopathy: anatomical, pathological and radiological correlation. Radiology, 146 (1): 1-9.

Robb, J. 1998. The interpretation of skeletal muscle sites: A statistical approach. International Journal of Osteoarchaeology, 8: 363-377.

Scheuer, L., S. Black. 2000. Developmental Juvenil Osteology. Elsevier Academic Press, Londres.

Sugiura, N. 1978. Further analysis of the data by Akaike's information criterion and the finite corrections. Communications in Statistics, A7: 13-26.

Vignati, M.A. 1960. El indigenado en la pcia. de Buenos Aires. Anales de la Comisión de Investigación Científica, I: 95-182.
Weiss, E. 2003. Understanding muscle markers: aggregation and construct validity. American Journal of Physical Anthropology, 121: 230-240.

Weiss, E. 2007. Muscle markers revisited: activity pattern reconstruction with controls in a central California amerind population. American Journal of Physical Anthropology, 133: 931-940.

Weiss, E., L. Corona y B. Schultz. 2012. Sex differences in musculoskeletal stress markers: problems with activity pattern reconstructions. International Journal of Osteoarchaeology, 22: 70-80.

Zar, J. 2010. Bioestatistical analysis. Pearson Education, Estados Unidos.

Zumwalt, A. 2006. The effect of endurance exercise on the morphology of muscle attachment sites. The Journal of Experimental Biology, 209: 444-454. 